\title{
Robust Focusing using Orientation Code Matching
}

\author{
Yuan $\mathrm{Li}^{*}$ Hidenori Takauji* Shun'ichi Kaneko* Takayuki Tanaka* ${ }^{*}$ Isao Ohmura ${ }^{+}$ \\ * System Sensing and Control lab,Graduate School of Information Science and Technology \\ Hokkaido University, Kita 14, Nishi 9, Kita-Ku, Sapporo, Japan \\ ${ }^{+}$Hokkaido Industrial Research Institute, Kita 19, Nishi 11, Kita-ku, Sapporo, Japan
}

Received 15th May 2008; accepted 12th May 2009

\begin{abstract}
This paper proposes a novel scheme for image focusing by introducing a new focus measure based on self-matching methods. A unique pencil-shaped profile is identified by comparing the similarity between all patterns extracted around the same position in each scene. Based on this profile, a new criterion function called Complementary Pencil Volume (hereafter CPV) is defined to evaluate focused or defocused scenes based on similarity rate of self-matching, which visually represents the volume of a pencil-shaped profile. Among matching methods, Orientation Code Matching (hereafter OCM) is recommended due to its invariance with regards to illumination and contrasts. Several experiments using a telecentric lens are implemented to demonstrate the efficiency of proposed measures. Outstandingly, comparing Orientation Code Matching-based (hereafter OCM-based) focus measure with conventional focus measures shows that OCM-based focus measure is robust against changes of illuminations and contrast. Using this method, depth is measured by comparing the focused and defocused region in the scenes both under high and low illumination conditions.
\end{abstract}

Key Words: Robustness, Focusing, Orientation Code Matching, Complementary Pencil Volume, Ill-conditioning, Depth Measurement.

\section{Introduction}

In the field of image processing and analysis, methods are proposed in order to enhance the quality of the image and the visualization, like focusing. A number of focus measures approaches, either active or passive, have been proposed and implemented in the literature. In this paper, passive focusing techniques which are more flexible and do not require calibration of the cameras are mainly concerened. As described in the literature $[9,4,13]$, when the lens of a camera is moving from one side to the other, the focus measure of a point of interest or window of a target object increases, reaches a maximum at the focus position and then decreases, corresponding to the sharpness of focus. An efficient criterion function is defined to measure the sharpness of focus of scene points at an unknown distance. Then a searching technique is needed to optimally locate the maximum of the criterion function. In this paper, a simple and unimodal focus measure based on self-matching methods is proposed. The maximum value of criterion function is easily searched. As an option, Fibonacci search algorithm, as demonstrated and suggested in [5], could also be efficient to locate the sharpest image of an object. For focusing, scaling and transform problems are significant when using a telescope lens. Normally

Correspondence to: <\{yuan,uji\}@ssc.ssi.ist.hokudai.ac.jp $>$

Recommended for acceptance by David Fofi and Ralph Seulin

ELCVIA ISSN:1577-5097

Published by Computer Vision Center / Universitat Autonoma de Barcelona 
camera calibration [20] is request before any operation. In this paper, instead of using a telescope lens, a simple telecentric lens is used. It will provide telecentricity of opticals [1]. A random dot pattern, which is composed of black and white pixels in equal proportions is also introduced. This random dot pattern contains rich textures for template matching. Without using a separate selecting algorithm, interested windows or points to be focused are randomly sampled for demonstration.

Some passive focus measures have been reported and compared. For example, to calculate the gradient magnitude [15], to use Bayes spectral entropy to construct the spectrum of an image [8], or to calculate the bimodal intensity histogram of entropy, to calculate the gray-level variance, etc., have been reported [15, 7]. Depth from focus has also been researched [12]. Unfortunately, these techniques are restricted regarding focusing conditions, such as ambient lighting, gain control and so on. In general, they require many pixels or wide regions in order to evaluate focus. Also, these passive focusing techniques may fail due to low contrast or illumination, such as dark, shadowy, obscured and cloudy conditions. The calculations require a certain degree of lighting or quantity of lights. These problems have not yet been resolved.

This paper therefore proposes a self-matching based focusing scheme utilizing OCM [18, 19]. In contrast to conventional focusing measures, this new scheme of focusing is not based on any evaluation of contrast or illumination, but instead on the evaluation of the similarity of any sub-image around a small neighborhood. Focus can be achieved through seeking the sharpest image by checking the similarities within the neighborhood. An original measurement of sharpness, CPV, which is based on a volume calculation, is proposed for robustly evaluating the significant peak profile in the similarity distribution. It measures the unique volume of a pencilshaped profile around the maximum similarity. This pencil-shaped volume is actually a visual representation of the sharpness of an image. In particular, this OCM-based focusing method can represent the steepest ascent orientation at each pixel in an image, instead of the brightness value. It is therefore robust against ill-conditioned images, and further, functions correctly in dark scenes [10]. All of these advantages are demonstrated and discussed. Additionally, depth images are measured and composed by using the proposed method.

In this paper, initially, the design of the new self-matching based focusing scheme is discussed; we also analyze the relationship between the pencil-shaped profile of self-matching similarity rates and sharpness of image scenes both in and out of focus; next, a number of convincing experiments performed under irregular contrast or illumination conditions are compared with conventional methods, Normalized Cross Correlation [3], Tenenbaum [15] and Sum-modulus-difference [6]. Depth images are tested using OCM-based CPV under different conditions. The conclusion comes last.

\section{Orientation Code Matching based self-matching}

Orientation Code has been firstly defined by F. Ullah and S. Kaneko, which is actually a virtual code created by quantizing the gradient angle of brightness of light at the corresponding pixel position in the gray images. More mathematically, the grey level of an image $I$ of pixel $(x, y)$ is denoted $I(x, y)$. And $\nabla I_{x}=\frac{\partial I}{\partial x}, \nabla I_{y}=\frac{\partial I}{\partial y}$ are respectively its horizontal and vertical derivatives. Then for any pixel $(i, j)$ of image, the orientation angle can be calculated as $\theta_{i, j}=\tan ^{-1}\left(\frac{\nabla I_{y}}{\nabla I_{x}}\right), \in[0,2 \pi]$. There are several filters can be utilized to calculate the gradient angle, the sobel filter is used in this study. Then the OC is defined through quantizing $\theta_{, j}$, by a constant width $\triangle_{\theta}$, as shown in formula 1 .

$$
c_{x, y}= \begin{cases}{\left[\frac{\theta_{x, y}}{\Delta_{\theta}}\right]} & \text { if }\left|\nabla I_{x}\right|+\left|\nabla I_{y}\right|>\Gamma \\ N=\frac{2 \pi}{\Delta_{\theta}} & \text { otherwise }\end{cases}
$$

where, $\Gamma$ is a threshold to suppress low contrast in the pixel neighborhood and noise. If $\Gamma$ value is set to be high, more useful information in image will be lost. While in this study, as discussed by Ullah, $\Gamma=10, \Delta_{\theta}=\frac{\pi}{8}$. The OC is obtained by quantizing $\theta_{i, j}$ by $\frac{\pi}{8}$ into $\mathrm{N}\left(=2 \pi / \frac{\pi}{8}=16\right)$. After orientation code transfer, a robust matching method called Orientation Code Matching is defined. For image $I$ and template $T$, the dissimilarity 


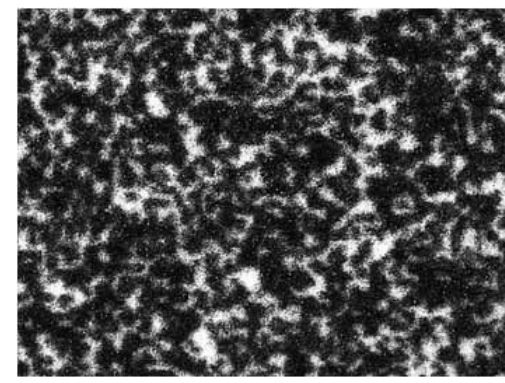

(a1) Focus scene

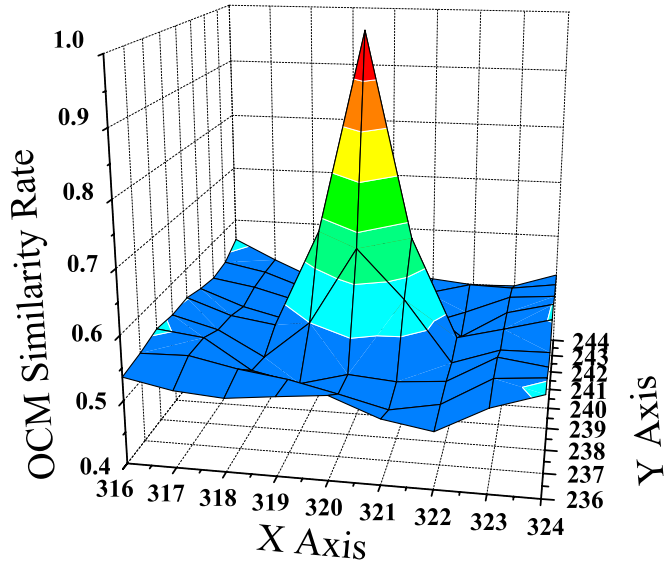

(b1) OCM Similarity Profile of (a1)

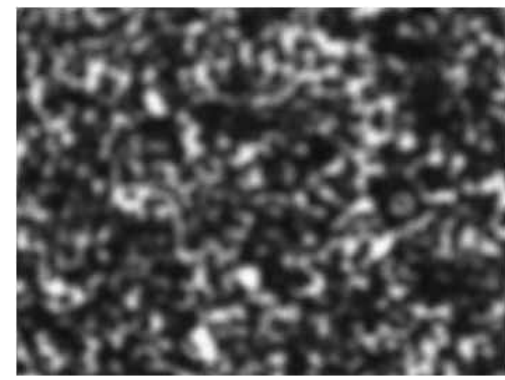

(a2) Defocus scene

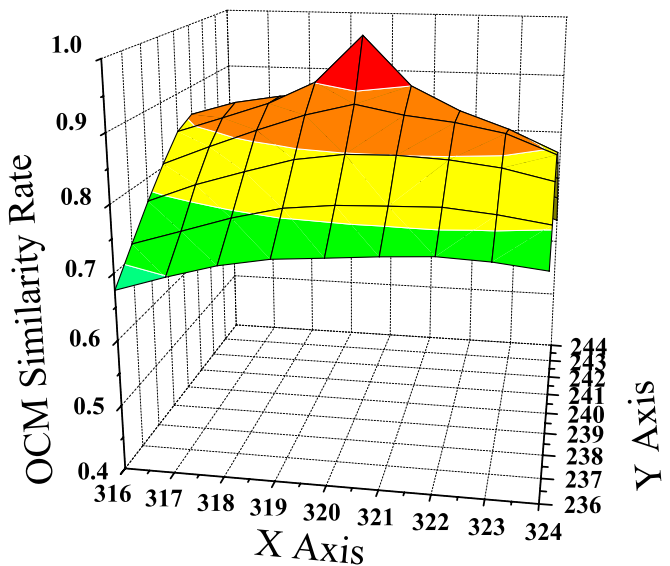

(b2) OCM Similarity Profile of (a2)

Figure 1: OCM similarity profiles of focus and defocus scenes.

rate is defined as:

$$
\begin{aligned}
D_{m, n} & =\frac{1}{M} \sum_{I_{m, n}} d\left(O_{I_{m, n}}(i, j), O_{T}(i, j)\right) \\
d(a, b) & =\left\{\begin{array}{cl}
\min \{|a-b|, N-|a-b|\} & \text { if } \quad a \neq N \text { and } b \neq N \\
\frac{N}{4} & \text { otherwise }
\end{array}\right.
\end{aligned}
$$

where $O_{I_{m, n}}$ and $O_{T}$ are the orientation code images of the reference image and the template respectively, $M$ is the size of the template and $d(a, b)$ is the error function based on absolute different criterion. Here, the orientation code is clockwise from 0 to 15 . For example, the $d(14,4)$ is 6 but not 10 in this case. When the contrast is lower than threshold, the $d(a, b)$ is equal to $\frac{N}{4}$. The matching similarity is defined as follow:

$$
S_{m, n}=1-D_{m, n}
$$

\section{Self-matching based focus measure}

In this section, focus measure based on self-matching methods are introduced. As known the original purpose of matching method was to search for similar template patterns in real world images. As an adaptation, a selfmatching is utilized to match any sub-image within the image itself. Fig.1(a1), (a2) shows two images, one is focused, the other is defocused, whose size are all $640 \times 480$. Although any part of an image scene can be 


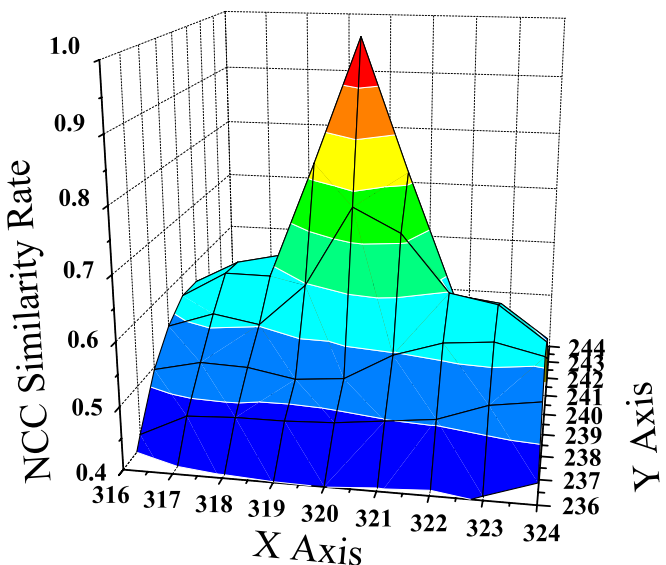

(a1) NCC similarity profile of Fig. 1(a1)

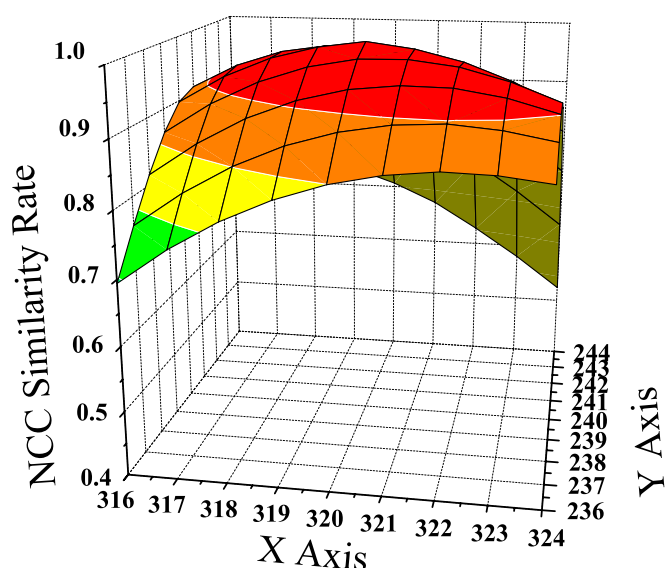

(a2) NCC similarity profile of Fig. 1(a2)

Figure 2: NCC similarity profiles of focus and defocus scenes.

selected as an interesting window, a sub-image, which center point is $(320,240)$ and size of $21 \times 21$ is utilized for illustration. The similarity of center point is the unit 1 , which is exactly matching position of the selected sub-image.

Figure. 1(b1) and Fig. 2(a1) shows self-matching similarity rate of OCM and NCC when scene is justin-focus. While Fig. 1(b2) and Fig. 2(a2) shows results of a defocus scene. In both the OCM and NCC profiles, at the top area near the unit, a special pencil-shaped profile is found and changes based on scene sharpness. When the image is sharp and focused, this pencil-shaped profile is obvious, and the similarity rate near the unit's neighborhood changes significantly. In contrast, when the image becomes blurry, it is more difficult to discriminate the neighborhood and the pencil-shaped profile becomes flatter. The volume of top area changes regards with the sharpness of image. When the image is just-in-focus, the volume becomes the minimum compared with defocus images. Additionally, compared with NCC, the similarity calculated using the OCM algorithm has very high sharpness or margin values around the correctly matching position regardless of illumination and contrast.

Figure. 1(b1) and Fig. 2(a1) shows self-matching similarity rate of OCM and NCC when scene is focused. While, Fig. 1(b2), (a2) shows results of a defocused scene. Both in OCM and NCC profiles, at the top area near the unit, a special pencil-shaped profile is found and changes based on scene sharpness. When the scene is sharp and focused, this pencil-shaped profile is obvious, and the similarity rate near the unit's neighborhood changes significantly. In contrast, when the scene becomes blurry, it is more difficult to discriminate the neighborhood and the pencil-shaped profile becomes flatter. Compared with NCC, the similarity calculated using the OCM algorithm has very high sharpness or margin values around the correctly matching position regardless of illumination and contrast. Experiments in Sec. 5 shows the efficiency of self-matching based focus measure based on both NCC and OCM. Additionally, the robustness of OCM-based focus measure against ill-conditioning scenes is also demonstrated.

\section{Complementary Pencil Volume criterion}

In Sec. 1, some conventional focus measures were introduced. However, these criteria are restricted with respect to illumination and the spatial requirements on objects. Also, the criteria are more complicated than expected. Therefore, the main topic in this section is how to effectively evaluate sharpness from similarity profiles in order to enhance changes in sharpness according to observations. In Sec. 3, we mentioned that the change of 


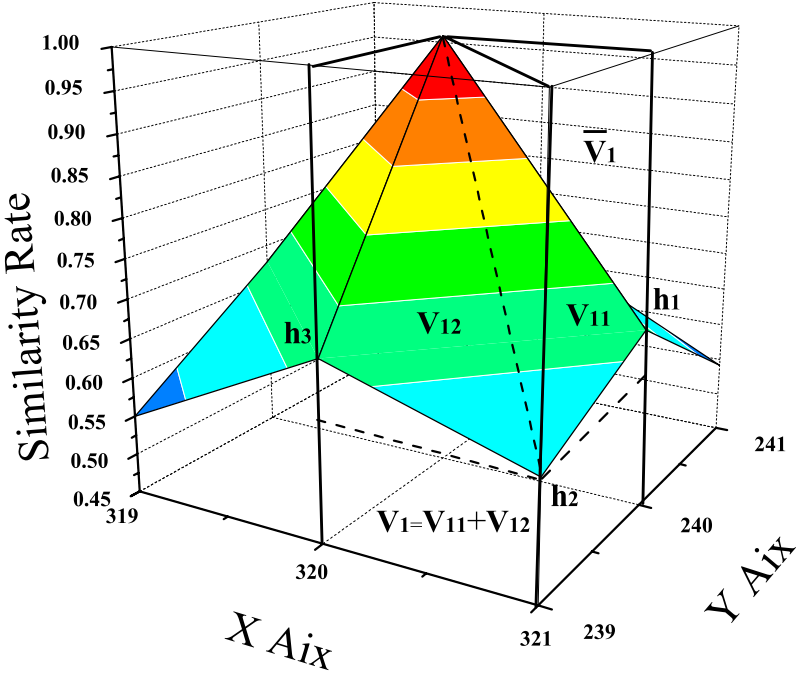

Figure 3: Pencil-shaped profile at the top of OCM similarity rate.

similarity has a different tendency or slope of pencil-shaped profile near the unite, depending on whether the image is focused or defocused. Therefore a volume calculation is proposed to quantitatively enhance changes in similarity profiles produced by self-matching in a $3 \times 3$ neighborhood. This is also the smallest neighborhood for template matching and efficient for proposed focus measure. Experiments using CPV criterion are in Sec. 5.

Figure. 3 shows a quarter of the pencil-shaped profile. The center point has the largest similarity, the unit. The vertices with heights $h_{1}, h_{2}$, and $h_{3}$ are the similarity rates of the neighborhood. According to simple geometry, the volume of this quarter, $V_{1}$, is composed from two simple triangular prisms, $V_{11}$ and $V_{12}$, whose volumes may be calculated as:

$$
\begin{aligned}
V_{1} & =V_{11}+V_{12} \\
& =\frac{1}{6} \times\left(1+h_{1}-2 h_{2}\right)+\frac{1}{6} \times\left(1+h_{3}-2 h_{2}\right) \\
& =\frac{1}{6} \times\left(2+h_{1}+h_{3}-4 h_{2}\right) .
\end{aligned}
$$

The CPV value $\bar{V}_{1}$ of $V_{1}$ is then calculated as:

$$
\begin{aligned}
\bar{V}_{1} & =\bar{V}_{11}+\bar{V}_{12} \\
& =\left(1-h_{2}\right)-V_{11}-V_{12} .
\end{aligned}
$$

The similar representations in the other quarters with indexes $i=2,3,4$ as follows:

$$
\begin{aligned}
\bar{V}_{i}= & \left(1-h_{2 i}\right) \\
& -\frac{1}{6} \times\left(2+h_{2 i-1}+h_{2 i+1}-4 h_{2 i}\right) .
\end{aligned}
$$

The total CPV of the pencil-shape is thus defined as:

$$
\bar{V}=\sum_{i=1}^{4} \bar{V}_{i}
$$




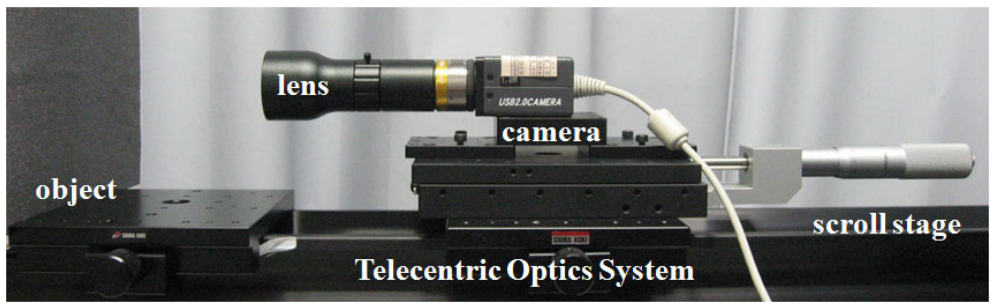

Figure 4: Experiments setup.

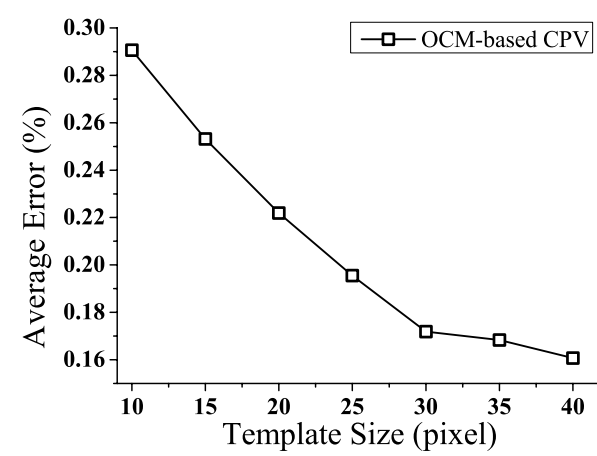

(a) Relationship between template size and depth error

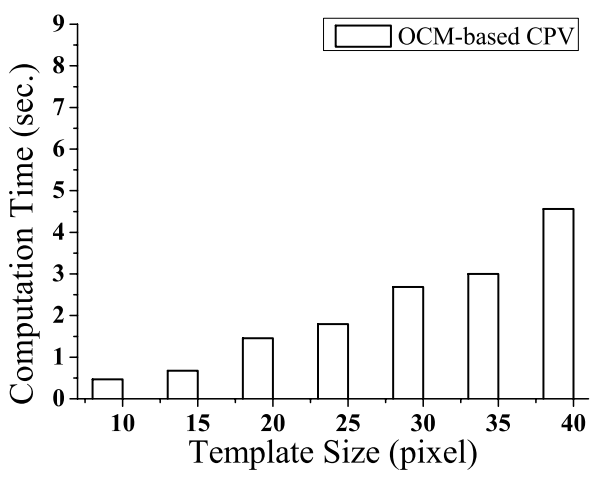

(b) Relationship between template size and computation time

Figure 5: Template size experiment results.

Although the image in focus has the smallest volume, after interpolation, the CPV has the largest value. This simple function can yield a sharp peak and the maximum of the criterion value. From these equations, it is concluded that the volume of the pencil-shaped profiles varies in relation to the similarity of self-matching alone. It is clear that shaper pencils corresponding to focused images have larger CPV values. Compared with other criteria, the CPV provides a simple but efficient method for finding the focused image quickly.

\section{Experiments}

The experiments setup is shown in Fig. 4. To evaluate the proposed focus measure, we use a planar object with a random dot pattern attached and take a sequence of images, systematically varying the lens distance, having the observed scene from defocused to focused and defocused again. A TECHSPEC ${ }^{T m}$ Silver Series HP telecentric measuring lens system with a mega-pixel USB camera made by Fortissimo is used to avoid transform and scale problems [16, 2]. The step is $0.1 \mathrm{~mm}$. Here, popular and conventional focus measures Tenenbaum [15] and Sum-modulus-difference (SMD) [6] are compared with focus measure based on NCC and OCM in high and low contrast conditions.

Before experiments, the self-matching template size is evaluated using OCM-based CPV. As shown in Fig. 5, as the size increasing the error of depth, which is calculated by OCM-based CPV, is decreasing, Meanwhile, this increase can also cost more computation time. As a compromise, size 21 is used in our experiments as the interested windows size of all interested points. This template size can be adapted considered on different experiments objects. 


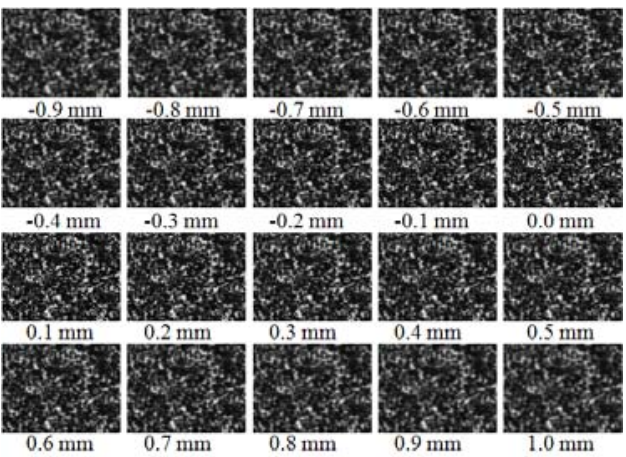

(a1) High contrast condition

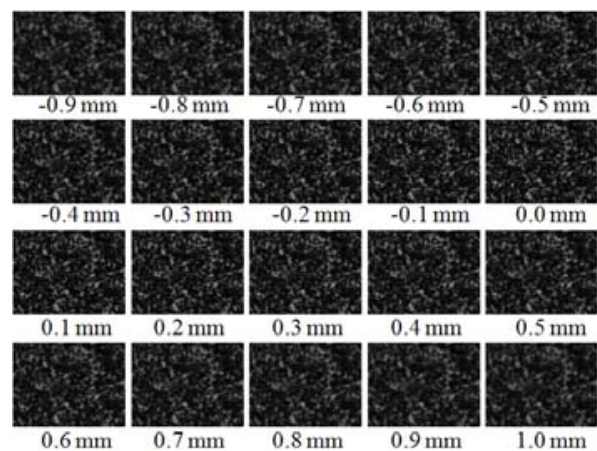

(a2) Low contrast condition

Figure 6: Image scenes under high and low contrast conditions.

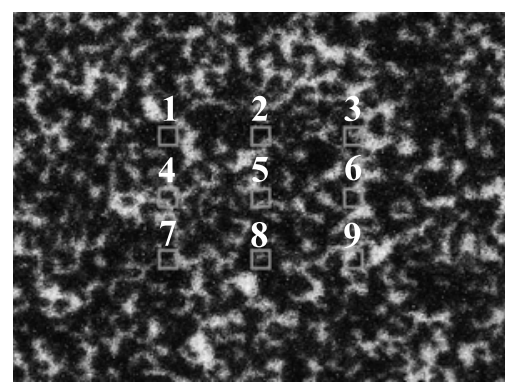

(a1) Focus scene under high contrast condition

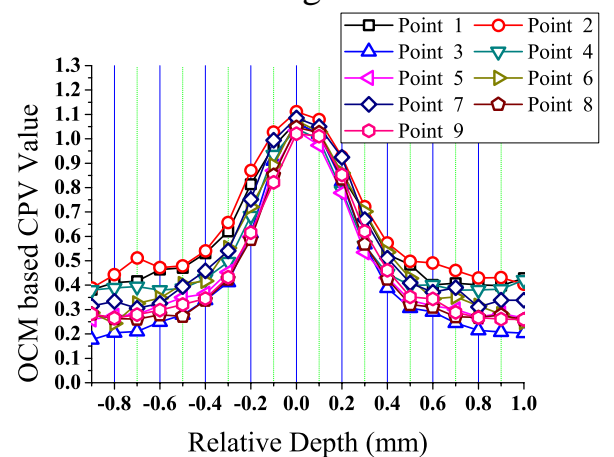

(b1) OCM-based CPV profiles under high contrast condition

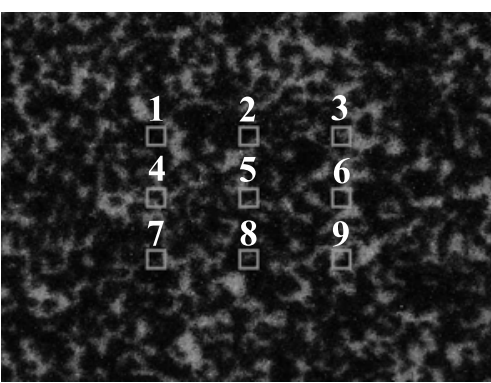

(a2) Focus scene under low contrast condition

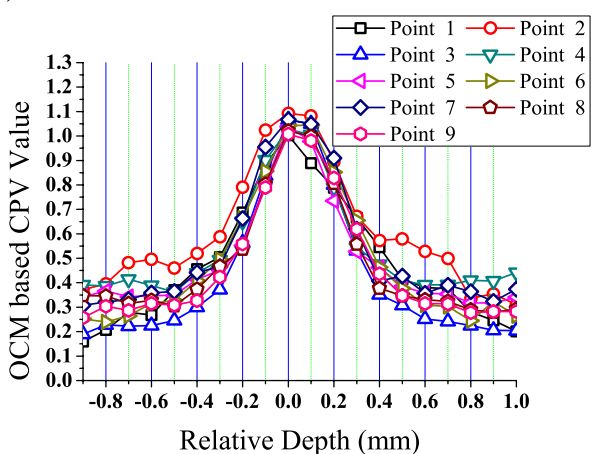

(b2) OCM-based CPV profiles under low contrast condition

Figure 7: OCM-based CPV profiles under high and low contrast conditions.

\subsection{Robustness to illumination and contrast using OCM-based CPV}

After we setting our experiments as described in former part, robustness of OCM-based CPV to illumination and contrast are implemented and discussed in this section. Fig. 6(a1), (a2) shows two image sequences of random dot pattern taken under high and low contrast individually by the Telecentric optical system. Each image is size of $640 \times 480$. Two image sequences are from out of focus to in focus and out of focus again. Only one original position $(0.0 \mathrm{~mm})$ is the focused position.

Figure. 7(a1), (a2) shows focused scenes of Fig. 6. Nine sampling points are evaluated. The generality of OCM-based profile is tested under both high and low contrast conditions. Results are shown in Fig. 7(b1), (b2). For each point of interest, one maximum, corresponding to the focused position is calculated. The profiles of all nine points are unimodal. The maximum of OCM-based CPV either under high or low contrast condition 
Table 1: OCM-based CPV results under different illumination conditions.

\begin{tabular}{c|c|c|c|c}
\hline $\begin{array}{c}\text { Illumination } \\
\text { (lux) }\end{array}$ & $\begin{array}{c}\text { Average } \\
\text { brightness }\end{array}$ & $\begin{array}{c}\text { Standard } \\
\text { deviation }\end{array}$ & $\begin{array}{c}\text { Average } \\
\text { gradient }\end{array}$ & $\begin{array}{c}\text { Average } \\
\text { error (mm) }\end{array}$ \\
\hline 500 & 11.46 & 10.16 & 22 & 0.02 \\
\hline 1000 & 23.17 & 21.44 & 57 & 0.01 \\
\hline 1500 & 34.40 & 31.97 & 89 & 0.02 \\
\hline 2000 & 47.05 & 44.48 & 130 & 0.01 \\
\hline 2500 & 59.99 & 55.72 & 166 & 0.02 \\
\hline 3000 & 67.55 & 61.68 & 185 & 0.02 \\
\hline
\end{tabular}

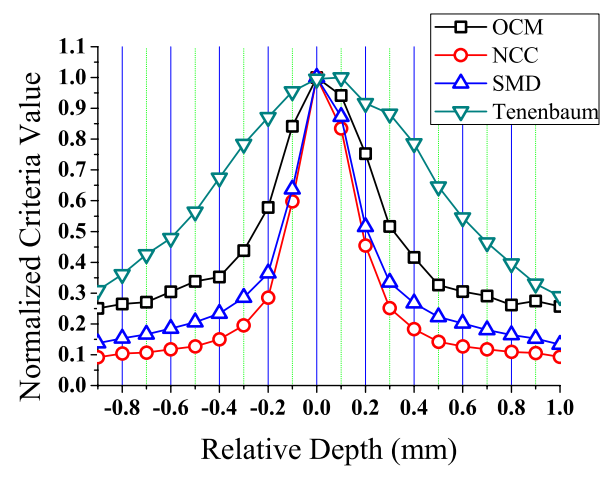

(a) High contrast condition

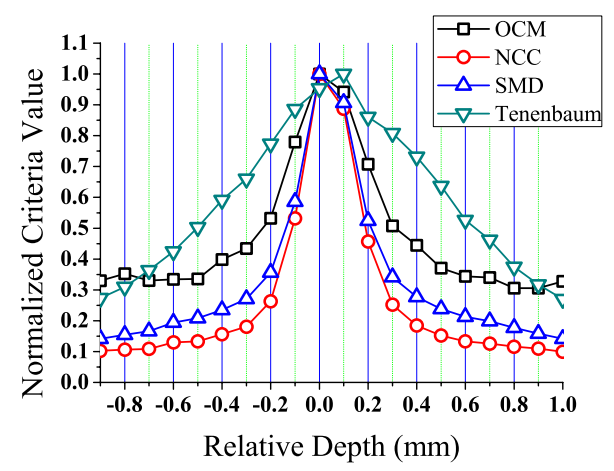

(b) Low contrast condition

Figure 8: Comparisons between normalized focusing criteria at the center points in Fig. 7(a).

is stable around the same value, because the orientation code is invariant. It calculates the gradient angle rather than gradient magnitude. OCM-based CPV is also evaluated under different illumination and contrast conditions. As shown in Table. 1, six conditions are estimated. The illumination is changed from 500 Lux to 3000 Lux. Average brightness, standard deviation and average gradient are changed based on different illumination and contrast conditions. With these changes, the average error of depth which is calculated using OCM-based CPV is stable from $0.01 \mathrm{~mm}$ to $0.02 \mathrm{~mm}$. From experiments above, it can be concluded that the OCM-based CPV is robust to illumination and contrast changes.

\subsection{Comparisons}

Comparisons OCM-based CPV and conventional methods, NCC-based CPV, Tenenbaum and SMD are carried out. Center points of Fig. 7(a) are estimated under different contrast situations. Normalized criteria results are shown in Fig. 8. All profiles of each focus measure were unimodal and had one maximum of criteria which is regarded as the focused scene. Results revealed that Tenenbaum shifted one step from the right position, while the others found right position successfully. When the illumination was maintained the same through focusing, NCC-based, OCM-based focus measures and SMD worked well even at relatively low illumination. Fig. 9 shows the un-normalized values of OCM-based CPV and SMD under high and low contrast conditions. As results shown, OCM-based CPV values of high contrast situations were similar to low contrast situations. In contrast, SMD values varied significantly from high contrast to low contrast. Both of them detected the one maximum of the focused scene correctly. Total computation time of all pixels $(640 \times 480=307200)$ using these four methods is estimated. As shown in Table. 2, OCM-based CPV is faster than NCC-based CPV and 


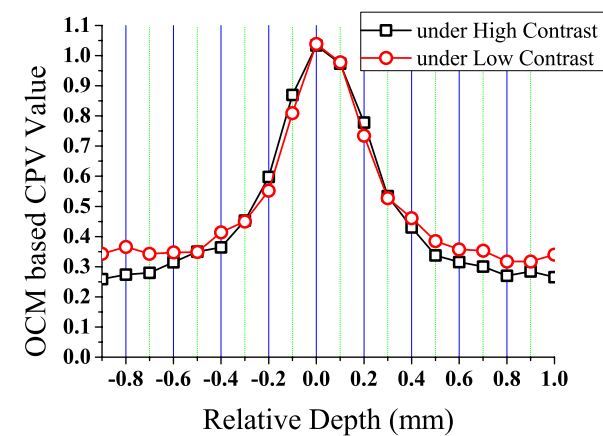

(a) OCM-based CPV profile

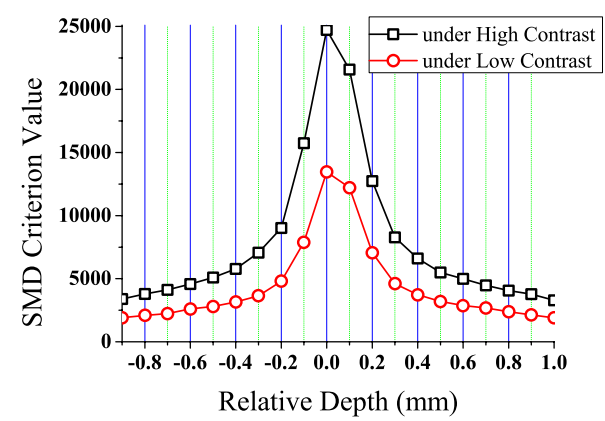

(b) SMD profile

Figure 9: Comparisons between OCM-based CPV and SMD under high and low contrast conditions.

Table 2: Computation time of different methods.

\begin{tabular}{c|c}
\hline Method & Computation Time (sec.) \\
\hline OCM & 137.86 \\
NCC & 251.78 \\
SMD & 17.96 \\
Tenenbaum & 175.64 \\
\hline
\end{tabular}

Tenenbaum but slower than SMD.

It is demonstrated that self-matching based focus measures are as good as SMD and better than Tenenbaum in former experiments. More delicate experiments have compared OCM-based focus measure with SMD. As shown in Fig. 10, two conditions are implemented and estimated. One is high-low contrast condition Fig. 10(a), the other is low-high contrast condition Fig. 10(b). At high-low condition, the focused scene has high contrast and two neighbors have low contrast, while the low-high condition has low contrast and two neighbors have high contrast.

When contrast became unstable, results illustrated in Fig. 10(d) reveal that, although SMD could find global maximum in a sequence, it has zigzag profile and is not unimodal. This zigzag profile could lead many failed local maximum values during image sequences. Especially, under low-high condition, global maximum was surely shifted, due to the different contrast from the neighborhood. Result of OCM shown in Fig. 10(c), was unimodal and had only one maximum corresponding to the scene in focus. This illustrates that OCM-based focusing measure is not only efficient to locate the image in focus, but also robust against illumination changes, that are common in our daily lives, like shadows or a cloudy day.

\section{Depth measurement}

Focusing has been considered as one of depth sources for computer vision. With the development of focusing measures, depth measurement is also been improved $[17,14,11]$. This section describes depth-from-focus using the proposed method. As one objective in the experiments is to ascertain whether the proposed method can reconstruct edges of severe depth changes in the image plane. The experimental setup is shown in Fig. 11. The optical system used was a TECHSPEC ${ }^{T M}$ Silver Series HP Telecentric Measuring Lens system with a mega-pixel USB camera which has no scaling and distortion affecting focusing results. The experimental objects were planar and had a random dot pattern attached to their surfaces. The distance between two planar 


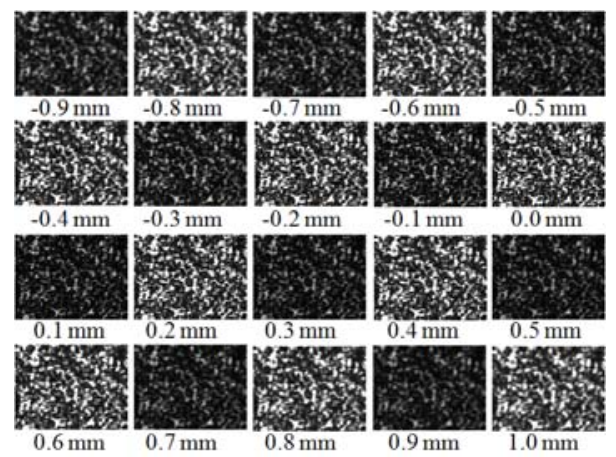

(a) High-Low contrast condition

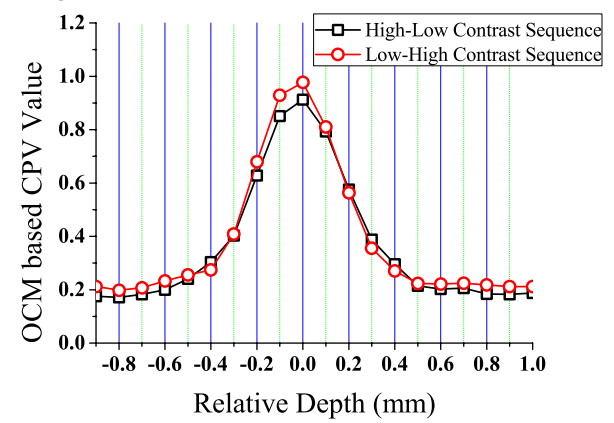

(c) OCM-based CPV profile of (a) \& (b)

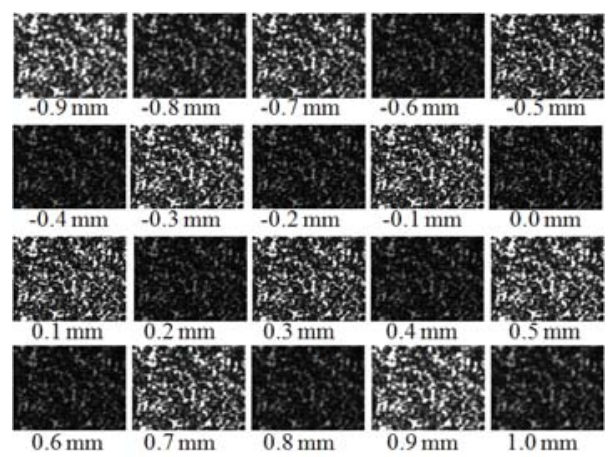

(b) Low-Hight contrast condition

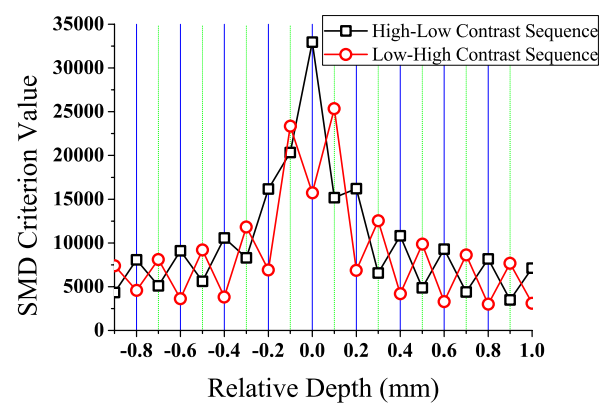

(d) SMD Profile of (a) \& (b)

Figure 10: Comparison between OCM-based CPV and SMD under different contrast conditions.

objects was $3.0 \mathrm{~mm}$ in the $\mathrm{Z}$ direction. They were fixed during experiments. The lens system was moved slightly by $0.1 \mathrm{~mm}$ in $\mathrm{Z}$ direction to take sequences of images. As illustrated in Fig. 11, there was an immediate or severe depth change at the edge of the front object from depth $\mathrm{Z}_{1}$ to $\mathrm{Z}_{2}$. Two planar objects belong to different planes of focus.

It is known that the optical and sensor configuration define a single plane of focus, such that every point on the plane is ideally focused onto only the image plane. As shown in Fig. 12(a), a slight change in the position of the lens relative to the image plane by replacing the lens causes the plane of focus to move in object space. The proposed OCM-based focusing measure is used to identify which plane of focus the object point belongs to and to measure the focusing distance of this plane. Then the depth of this object point is equal to the focusing distance. The relationship between the plane of focus and the depth in the $\mathrm{Z}$ direction is illustrated in Fig. 12(b). It is clear that all points sharing the same plane of focus have the same depth measurement.

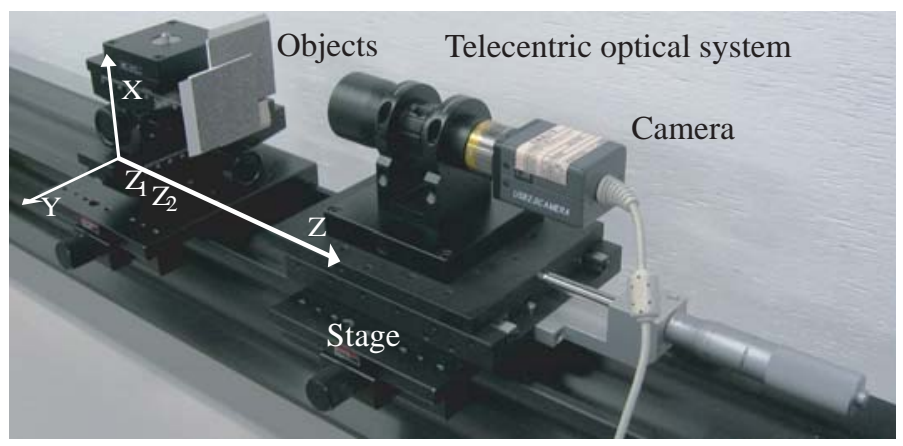

Figure 11: Depth measurement experiment setup. 


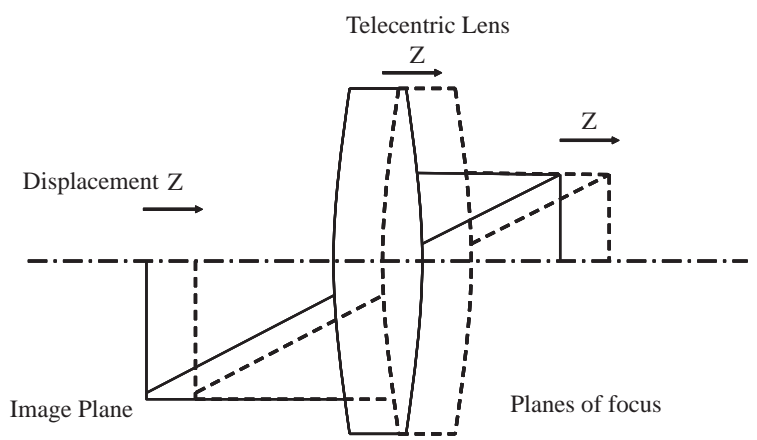

(a) Telecentric lens focusing geometry

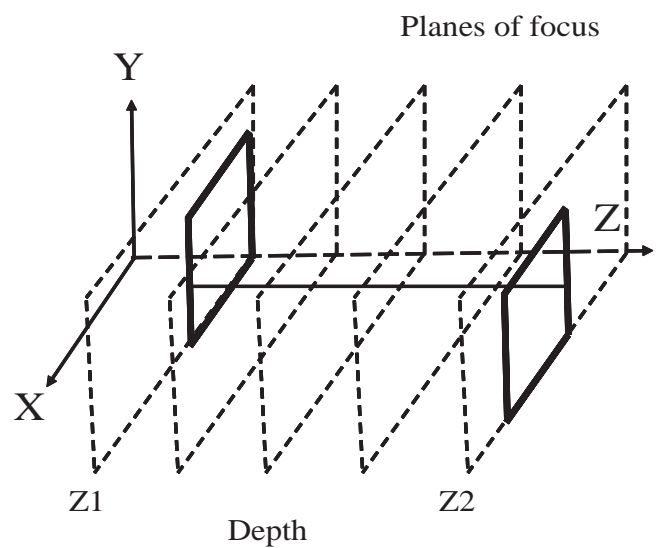

(b) Relationship between plane of focus and depth $\mathrm{Z}$

Figure 12: Depth measurement illustration.

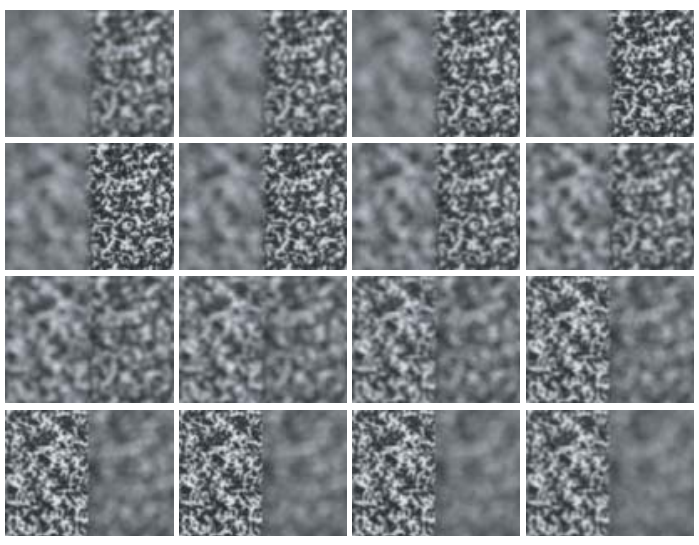

(a) Scenes under normal conditions

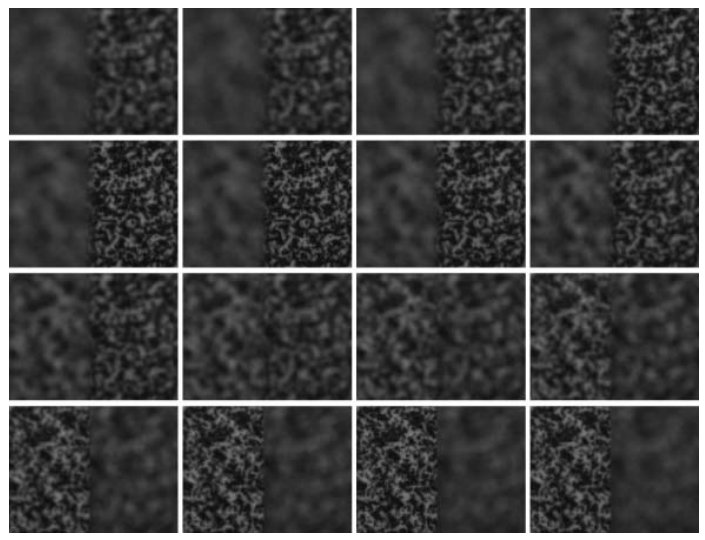

(b) Scenes under dark conditions

Figure 13: Sequences of images taken with the telecentric optical system.

Measured sequences of images are shown in Fig. 13(a) and (b) under normal and dark conditions individually. When using a telecentric lens, each image is divided into two half segments whose sharpness of focus is regarded as indicating a different focusing distance or displacement of two planar objects. A slight change of displacement of the telecentric lens system caused the plane of focus to move in the object space. However only one segment was focused in image space in a certain displacement. In the measured image, there is a center edge between the two regions of $3.0 \mathrm{~mm}$ displacement.

As previously demonstrated, the proposed OCM-based robust focusing method can quantitatively calculate the quality of image focus. Only one maximum CPV corresponds to the displacement of the just-in-focus image. The method is also robust against ill-conditioning. For each point of interest, OCM-based focusing is able to find the best planes of focus among the quantized depths based on these profiles. The depth images may therefore be composed using the maximum value as an index to calculate the depth of the planes of focus.

Results of depth reconstruction are shown in Fig. 14. The depth images of two objects are constructed in normal and dark conditions, and by three different focusing approaches: Contrast Detection, NCC-based, and OCM-based focusing. The depth is measured at all object points to visually represent the depth information of 


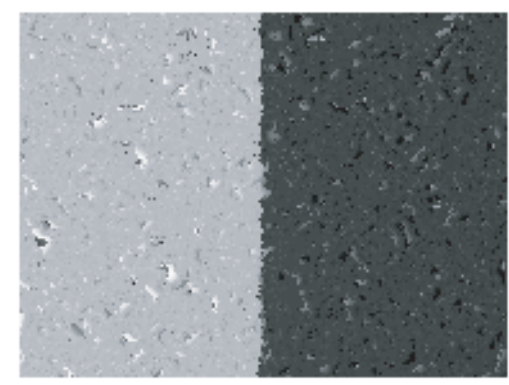

(a) Depth image for Contrast Detection

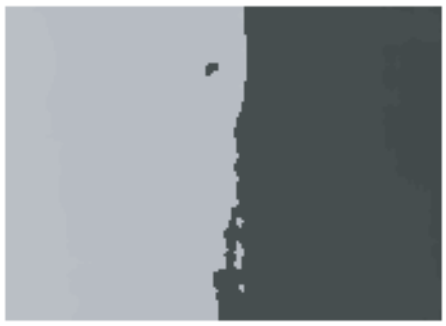

(c) Depth image under NCC-based focusing

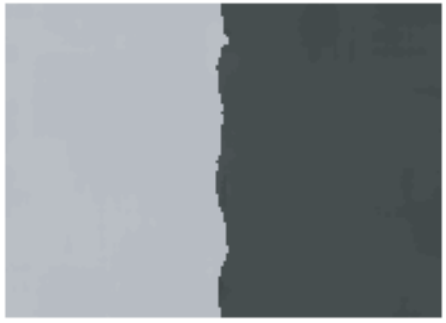

(e) Depth image under OCM-based focusing

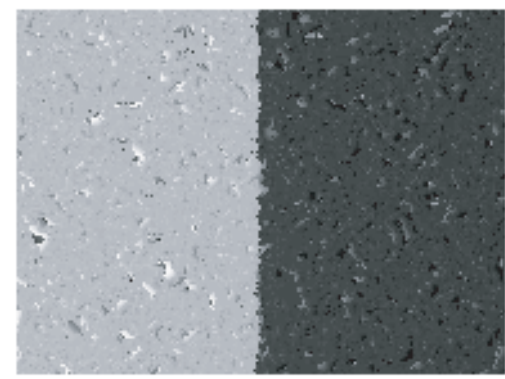

(b) Depth image under Contrast Detection in dark conditions

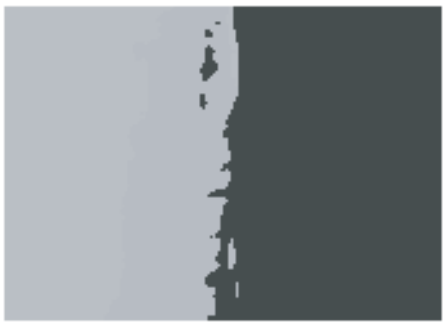

(d) Depth image under NCC-based focusing in dark conditions

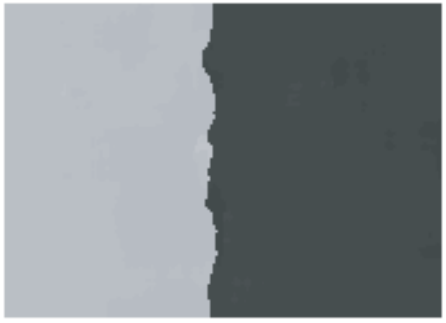

(f) Depth image under OCM-based focusing in dark conditions

Figure 14: Depth images and comparisons.

the objects. When the depth increases, the brightness of images varies from dark to bright. Depth reconstruction on the edge is highly outlined and compared in this experiments.

As shown in Fig. 14(a) and (b), the Contract Detection approach failed to calculate the depth even at the points on both planar objects. Many mistake black or white spots appeared at both region. A zigzag line was constructed on the center edge. The depth images were coarse and rough. Same phenomena happen under the dark condition as well. Fig. 14(c) and (d) show the results of NCC-based focusing under normal and dark conditions separately. Compared with the Contrast Detection, NCC-based focusing more successfully calculated the depth of the two planar objects almost positions. Few mistake spots appeared. However, the results on the center edge were rather disappointing. It was clear that the center edge shifted to the left of the depth image under both normal and dark conditions, and was even worse under later conditions. NCC-based focusing could succeed on smooth changes of depth, but failed when depth changes sharply, such as the center edge in Fig. 13. In contrast to these two focusing methods, OCM-based focusing, shown in Figs. 14(e) and (f), could not only measure the depth of objects at different distances, but also had better results at the center edge of sharp depth changes. It was smoother and neater than the results of the other two methods. There was a significant change of brightness when the depth changes. The depth images were more delicate and refined. 
The OCM-based method also succeeded under dark conditions. The characteristic of robustness against the brightness or illumination change was thus demonstrated again.

The different results obtained with these three methods at the center edge of depth changes showed that OCM-based focusing had higher spatial resolution than the other methods. As defined in Fig. 3, the criterion function CPV evaluates a $3 \times 3$ neighborhood, which is the smallest domain or region for estimating the sharpness of identical points of interest in different positions. This smallest domain is sufficient to calculate the depth comprehensively, while the Contrast Detection bases on four neighboring pixels. Although, the NCCbased method was attempted on the same domain, the results in Fig. 14 revealed that OCM-based focusing achieved the best and most precise results of all the methods and had a high special resolution.

\section{Conclusions}

A new focus measure was proposed by analyzing the different between similarity rates of self-matching. Specifically, a novel and robust focusing method based on Orientation Code Matching was designed and compared with conventional methods. A unique pencil-shaped profile is visually and mathematically generated depending on the sharpness of focus. A new criterion, Complementary Pencil Volume was proposed in order to achieve focusing. Experiments have proved that self-matching focus measure can successfully find a focused image in sequences. Importantly, OCM-based focus measure is robust and effective, especially in low contrast situations. Object depth measurement by OCM-based focusing were demonstrated in this paper. Experiments revealed that the proposed method achieved better and more precise results than some of conventional methods.

\section{References}

[1] M. Born and E. Wold, Principles of Optics, Pergamon Press, Oxford, Sixth Edition, 1980.

[2] 1/2 inch CMOS camera, Fortissimo Corporation, http://www.ff-net.ne.jp/ffhome/cmos130.html, 2000.

[3] R. C. Gonzalez and R. E. Woods, Digital Image Processing (third edition) Reading Massachusetts, Addison-Wesley, 1992.

[4] B. K. P. Horn, ’Focusing", MIT Artificial Intelligence Laboratory MEMO NO. 160, 1968.

[5] S.M. Johnson, "Optimal search for a maximum is Fibonaccian", RAND Corp. Rept. P-856, Santa Monica, Calif., 1956.

[6] R. A. Jarvis, "Focus optimization criteria for computer image processing", Microscope, Vol. 24, NO. 2, pp. 163-180, 1976.

[7] R. A. Jarvis, "A perspective on range finding techniques for computer vision", IEEE Trans. Pattern Analysis and Machine Intelligence, Vol. PAMI-5, Number 2, pp. 122-139, 1983.

[8] M. Kristan, J. Pers, M. Perse and S. Kovacic, "A Bayes-spectral-entropy-based measure of camera focus using a discrete cosine transform”, Pattern Recognition Letters, Vol.27, 23, pp. 1431-1439, 2006.

[9] E. Krotkov, ”Focusing", International Journal of Computer Vision Vol.1, 3, pp. 223-237, 1987.

[10] Y. Li, I. Ohmura, H. Takauji, S. Kaneko and T. Tanaka, "Robust focusing by orientation code matching and its application to depth reconstruction", The 8th International Conference on Quality Control by Artificial Vision, Proc.SPIE, 6356, (2007) 635610-1-10-8.

[11] G. Ligthart and F. Groen, "A comparision of different autofocus algorithms", International Conference on Pattern Recognition, pp. 597-600, 1982. 
[12] S. K. Nayar and Y. Nakagawa, "Shape from focus:an effective approach for rough surfaces", IEEE International Conference on Robotics and Automation, Vol.2, pp. 218-225, 1990.

[13] M. Subbarao, T. Chio and A. Nikzad, "Focusing techniques", Optical Engineering 32(11), pp. 2824-2836, 1993.

[14] M. Subbarao, T. Choi, "A new method for shape from focus", SPIE International Symposium on Optical Tools for Manufacturing and Advanced Automation, Vol. 2006(08), 1993.

[15] J. F. Schlag, A.C. Sanderson, C.P. Neuman, and F.C. Wimberly, 'Implementation of automatic focusing algorithms for a computer vision system with camera control", Tech. report CMU-RI-TR-83-14, Robotics Institute, Carnegie Mellon University, 1983.

[16] 1.0X Techspec Silver Series Telecentric Lens, Edmund Optics Inc.

[17] A. Pentland, "Depth of scene from depth of field", Proceedings of Image Understanding Workshop, pp.253-259, 1982.

[18] F. Ullah, S. Kaneko and S. Igarashi, "Orientation code matching for robust object search", IEICE Trans. On Inf.\& Syst., Vol.E84-D, No. August, 2001.

[19] F. Ullah and S. Kaneko, "Using orientation codes for rotation invariant template matching", Pattern Recognition, Vol.37, pp. 201-209, 2004.

[20] R.G Willson and S.A. Shafer, "Modeling and calibration of automated zoom lenses", Technical report CMU-RI-TR-94-03, Robotics Institute, Carnegie Mello Univ., Pittsburgh, Pa., Jan.1994. 